\title{
¿Pueden los bosques tropicales resistir o recuperarse del impacto de los huracanes?
}

\author{
Georgina García-Méndez, Víctor J. Jaramillo, Angelina Martínez-Yrízar, Manuel \\ Maass, José Sarukhán, Maribel Nava-Mendoza, Raúl Ahedo y Salvador Araiza
}

\section{Resumen}

El estudio de largo plazo (35 años) sobre los procesos ecológicos funcionales del bosque tropical caducifolio en la región de Chamela, Jalisco, en la costa del Océano Pacífico, ha sido una herramienta científica muy útil para entender las propiedades del ecosistema y su variabilidad natural. Nos ha permitido documentar, por ejemplo, las alteraciones del bosque en términos de la pérdida de suelo por erosión, y la caída de hojas y árboles debido a huracanes originados tanto en la costa del Pacífico como en la costa Atlántica. Ahora sabemos que el bosque de Chamela tiene alta capacidad de recuperación ante el impacto de los huracanes, pero que dicha recuperación depende en gran medida de la cantidad de lluvia que cae de manera posterior a los mismos. Hemos podido documentar que existe una gran incertidumbre en la cantidad y en la distribución estacional de la lluvia, y cómo es que ésta determina si el bosque es capaz de recuperarse y con qué rapidez. Proponemos que es necesario incorporar dicha incertidumbre tanto en el diseño de estrategias para lograr su conservación, como en estrategias adaptativas y de manejo de ecosistemas que disminuyan la vulnerabilidad de los habitantes de las regiones del trópico estacionalmente seco, expuesto a los impactos de estos eventos.

Palabras clave: resiliencia, huracanes, variabilidad climática, bosque tropical caducifolio, Chamela, proyectos de largo plazo.

\section{CAN TROPICAL FORESTS RESIST OR RECOVER FROM HURRICANE IMPACTS?}

\begin{abstract}
The long-term study (35 years) on the functional ecological processes of the tropical deciduous forest of the Chamela region, Jalisco, on the Pacific coast of México, has been a very useful scientific tool for the understanding of the ecosystem processes and their natural variability. It has allowed us to document, for example, the degree of forest disruption in terms of soil loss due to erosion, and the loss of the canopy and tree fall due to hurricanes originating both in the Pacific and the Atlantic coasts. We now know that the Chamela forest has a high capacity to recover from the impact of hurricanes, but such recovery depends on the amount of precipitation occurring after their impact. We have been able to document that the uncertainties in the amount and seasonal distribution of rainfall determine whether and how fast the forest is capable of recovery after disturbance. We propose that there is a need to incorporate such uncertainty in the design of forest conservation, adaptive and management strategies, to reduce the vulnerability of the communities in seasonally dry tropical regions exposed to the impacts of these extreme events.
\end{abstract}

Keywords: resilience, hurricanes, climatic variability, tropical deciduous forest, Chamela, long-term projects.

Recepción: 30/07/2019. Aceptación: 20/05/2020. Dol: http://doi.org/10.22201/cuaieed.16076079e.2020.21.5.5 
"¿Pueden los bosques tropicales resistir o recuperarse del impacto de los huracanes?"

Georgina García-Méndez, Victor J. Jaramillo, Angelina Martínez-Yrízar,

Manuel Maass, José Sarukhán, Maribel Nava-Mendoza, Raúl Ahedo y Salvador Araiza

Vol. 21, Núm. 5, septiembre-octubre 2020

Revista Digital Universitaria

\section{Georgina García-Méndez}

gmendez@ecologia.unam.mx

http://orcid.org/0000-0002-4458-1551

geo.garcia.mendez@gmail.com

Ha desarrollado su carrera profesional como técnica académica en el Instituto de Ecología de la Universidad Nacional Autónoma de México (UNAM), participando en diversos proyectos relacionados con el estudio de la estructura y funcionamiento de un ecosistema tropical estacional en Chamela, Jalisco, así como la evaluación del impacto que diferentes técnicas de manejo de recursos tienen en la productividad primaria y el balance nutricional de dicho bosque. También participó en el proyecto conjunto entre el Centro de Ecología, la Universidad de Stanford y el Ames Research Center de la NASA, destinado a medir las emisiones de óxido nitroso en dos sitios de selva y en un pastizal en Chamela, Jalisco. Desde 1999 ha apoyado las actividades académicas del Dr. Sarukhán en el Instituto de Ecología, entre las que destacan el apoyo en la coordinación de las actividades académicas de la Maestría en Restauración en el Posgrado en Ciencias Biológicas de la unAm hasta 2010, y en la coordinación de los cursos obligatorios del campo de conocimiento de Restauración Ambiental del Posgrado en Ciencias de la Sostenibilidad de la unAm hasta la actualidad. Participó en el proceso de elaboración de la obra Capital Natural de México, publicado por la Comisión Nacional para el Conocimiento y Uso de la Biodiversidad (CONABIO), como integrante del secretariado. También formó parte del equipo que elaboró la Estrategia Nacional sobre biodiversidad de México y su Plan de Acción 2016-2030, bajo la supervisión del Dr. José Sarukhán.

\section{Víctor J. Jaramillo}

Su línea principal de investigación es la Biogeoquímica de Ecosistemas Tropicales, con énfasis en la dinámica del carbono, nitrógeno y fósforo en el trópico seco. Ha utilizado enfoques tanto experimentales como de monitoreo de largo plazo y en una variedad de escalas espaciotemporales, que van desde procesos microbianos en el suelo, procesos ecofisiológicos en árboles, hasta procesos ecosistémicos en escala de pequeñas cuencas hidrológicas. Pertenece al grupo de investigación ecosistémica de largo plazo conocido informalmente como Grupo Cuencas, con el que ha colaborado durante 25 años en el proyecto "Estructura y funcionamiento de un ecosistema tropical seco en Chamela, Jalisco", forma parte del Grupo Chamela de la Red Mexicana de Estudios a Largo Plazo (Red MeX-LTER), de la Red de Socioecosistemas del CONACYT, y de una red internacional para el estudio del nitrógeno en Latinoamérica conocida como Nnet, en la cual participan investigadores de siete países de América.

\section{Angelina Martínez-Yrízar}

Es ecóloga de ecosistemas del Instituto de Ecología, unAm, donde investiga, con un enfoque de largo plazo, la productividad primaria, la biomasa y la descomposición de hojarasca en ecosistemas limitados por agua, principalmente del bosque tropical seco y del Desierto Sonorense en México. Analiza la resiliencia del bosque tropical seco a heladas severas en el límite norte de su distribución en América y a los huracanes en la reserva de Chamela en la costa del Pacífico. También estudia la mortandad inducida por sequía en plántulas de especies mexicanas de pino, para entender los mecanismos de resiliencia a eventos climáticos extremos como sequía y olas de calor. Otros tópicos de su investigación incluyen la extracción selectiva de recursos forestales no maderables y el análisis de su efecto en la biodiversidad del bosque tropical seco y los mecanismos de regeneración natural. También realiza investigación desde la perspectiva biocultural de los grupos indígenas de Sonora, analizando la problemática de acceso al agua y cómo afectan diferentes sistemas de manejo y uso de los recursos la biodiversidad y la conservación dentro y fuera de los territorios indígenas. Es miembro del Sistema Nacional de Investigadores desde 1993, miembro del Grupo Chamela de la Red Mex-LTer y de las Redes Temáticas "Etnoecología y Patrimonio Biocultural de México" y "SocioEcosistemas y Sustentabilidad" del conACYT. 
"¿Pueden los bosques tropicales resistir o recuperarse del impacto de los huracanes?"

Georgina García-Méndez, Victor J. Jaramillo, Angelina Martínez-Yrízar,

Manuel Maass, José Sarukhán, Maribel Nava-Mendoza, Raúl Ahedo y Salvador Araiza

Vol. 21, Núm. 5, septiembre-octubre 2020

Revista Digital Universitaria

\section{Manuel Maass}

Sus líneas de investigación incluyen: Redes Académicas de Investigación Científica; Investigación Ecológica de Largo Plazo (LTER); Investigación transdisciplinaria para el manejo de Socioecosistemas; Ecología de Ecosistemas Tropicales Secos; Manejo Integrado de Cuencas Hidrológicas; y Ecología Funcional (Eco-hidrología, Energética y Biogeoquímica). Ha concentrado su actividad académica al participar por más de 35 años en un estudio de largo plazo sobre "Estructura y funcionamiento de un ecosistema tropical seco en Chamela, Jalisco". Fue miembro fundador y primer Coordinador Nacional de la Red Mex-LTeR, así como miembro del Comité Directivo de la Asociación Internacional de Ecología (INTECOL) y electo presidente (Chair) de la Red Internacional de Investigaciones Ecológicas a Largo Plazo (ILTER) para el período 2012-2016. Participó en el proceso de establecimiento de campus foráneos de la UNAM, y fue Coordinador Administrativo del Campus Morelia durante sus 3 primeros años de operación. Ha ocupado puestos de representación académica en la UNAM (CTIC, CAABQYS, UACPYP y Consejo Universitario). Participó en la Junta de Gobierno del Instituto de Ecología, A.C. (INECOL) y en diversas Comisiones Dictaminadoras de instituciones académicas dentro y fuera de la UNAM. Actualmente coordina los esfuerzos para crear un Observatorio Nacional para la Sustentabilidad Socioecosistémica (ONSSES) con apoyo del CONACYT (programa: Atención a Problemas Nacionales).

\section{José Sarukhán}

Sus líneas de investigación son la ecología de poblaciones y sistemas, la ecología global, la biodiversidad y su evolución, así como aspectos de educación superior, ciencia y tecnología ligadas al desarrollo. Fue promotor del principal grupo de investigación ecológica en México y del ahora Instituto de Ecología de la unAm, además de investigador fundador del proyecto de largo plazo "Estructura y funcionamiento de un ecosistema tropical seco en Chamela, Jalisco". En 1991 propusó al Gobierno Federal la creación de la Comisión Nacional para el Conocimiento y Uso de la Biodiversidad (conABio), donde fungió como Coordinador Nacional y desde 2010 dirije a la conABIO en sus labores diarias. Es miembro de El Colegio Nacional, de la Third World Academy of Sciences, de la Academia de Ciencias de California, de la Academia Europea de Ciencias, de la National Academy of Sciences (USA), de la Royal Society del Reino Unido y de la American Academy of Arts and Sciences. Ha sido miembro de la Junta de Directores del World Resources Institute, Washington, D.C. (2000-2009); de las Juntas de Gobierno del El Colegio de México (2000-2005) y de la Universidad Veracruzana (2009-2014). Ha recibido diversos premios y reconocimientos nacionales e internacionales y ha publicado más de 190 trabajos científicos y varios libros, entre ellos: tres ediciones de Árboles Tropicales de México, ocho ediciones de Las Musas de Darwin y el Manual de Malezas del Valle de México. Coordinó la publicación de la obra Capital natural de México conformada por cinco volúmenes. Los tres primeros volúmenes, comprenden 45 capítulos, en los que participaron 96 revisores externos y 648 autores provenientes de 227 instituciones, tanto académicas como gubernamentales y de organizaciones civiles de la mayor parte de las entidades de la República.

\section{Maribel Nava-Mendoza}

Participa brindando asesoría técnica en el laboratorio de Biogeoquímica para el grupo de investigación de largo plazo en el bosque tropical seco de Chamela, y realizando determinaciones químicas (carbono, nitrógeno y fósforo) en material vegetal, suelo y agua. Ha impartido 45 cursos de licenciatura (40 semestrales y 5 cursos cortos tanto en la Universidad Michoacana de San Nicolás de Hidalgo como en la Escuela Nacional de Estudios Superiores, unAm en Morelia). Además, ha impartido cursos de capacitación técnica sobre el uso de la coulometría y la colorimetría como herramientas para la determinación de elementos. Ha recibido agradecimientos en 55 documentos científicos que incluyen artículos en revistas indizadas, tesis, memorias de congresos y capítulos de libro y la medalla "Sor Juna Inés de la Cruz" otorgada por la unam. 


\section{Raúl Ahedo Hernández}

Brinda apoyo técnico-logístico al laboratorio del Dr. Manuel Maass (IIES-UNAM), además participa en el grupo de investigación de largo plazo "Estructura y funcionamiento de un ecosistema tropical seco en Chamela, Jalisco", como técnico responsable de la sistematización de datos hidrológicos (lluvia y escorrentía) y del trabajo de campo para el monitoreo del crecimiento y la mortalidad de árboles, los cuales llevan monitoreándose por más de 30 años. Es coautor de 4 artículos de investigación indexados y su apoyo técnico ha sido agradecido en 77 productos de investigación y en 54 tesis.

\section{Salvador Araiza}

Técnico de campo para el proyecto de largo plazo "Estructura y funcionamiento de un ecosistema tropical seco en Chamela, Jalisco", especializado en la recolección y manejo de muestras vegetales, suelos y agua, así como en la captura de datos micrometeorológicos y ambientales. Ha recibido agradecimientos en 40 artículos publicados en revistas arbitradas y en 8 capítulos de libro, y he sido coautor en 3 trabajos presentados en reuniones y en 9 carteles en congresos. Además, su apoyo técnico ha sido reconocido en 41 tesis de licenciatura, maestría y doctorado. 


\section{Introducción}

Antes de adentrarnos en el tema, es preciso explicar que la tendencia actual de calentamiento de la atmósfera del planeta está provocando también un aumento en la temperatura de los océanos, que es donde se originan los huracanes. Y como es bien sabido, a mayor temperatura, mayor es la energía disponible para su formación. Por ello, se predice que, con el calentamiento global, motor del cambio climático, los huracanes se volverán cada vez más intensos, con vientos más veloces y con mayor capacidad destructiva. Es por ello que el estudio del impacto de los huracanes, no sólo en nuestro país, sino en todo el mundo, se ha convertido en un tema de gran importancia desde las perspectivas biológica, ecológica, económica y social.

Imagen 1. Incidencia de huracanes en México de acuerdo con SEMARNAT, 2018. Fuente: Semarnat. Informe de la Situación del Medio Ambiente en México. Compendio de Estadísticas Ambientales. Indicadores Clave,

de Desempeño Ambiental y de Crecimiento Verde. Edición 2015. Semarnat. México. 2016.

\section{Nuestro país es vulnerable ante el cambio climático}

México tiene características geográficas que lo colocan como uno de los países más vulnerables a los efectos del cambio climático (ENCC, 2013; PECC 2014-2018), entre los que se encuentran el aumento proyectado en la intensidad de los ciclones tropicales en años venideros (SEMARNAT-INECC, 2012). Su localización entre dos océanos, su latitud y relieve accidentado y montañoso, lo exponen a diferentes fenómenos hidrometeorológicos, como los huracanes (ENCC, 2013; PECC 2014-2018), que pueden presentarse tanto en los litorales del Golfo de México y del Océano Atlántico, como en el litoral del Océano Pacífico. Prueba de ello son los 22 huracanes de gran intensidad (categorías 3, 4 y 5 de la escala de vientos de SaffirSimpson) que han hecho tierra en distintos puntos de las costas del país entre 1970 y 2017 (SEMARNAT, 2018, ver Imagen 1). Ciertamente, los más afectados son los ecosistemas costeros y sus habitantes; sin embargo, sus efectos se extienden en ocasiones a muchas otras regiones del país situadas tierra adentro. Si la intensidad de los huracanes aumenta significa que diversos ecosistemas estarán expuestos a eventos cada vez más devastadores, poniendo en riesgo tanto a las especies de plantas y 
Imagen 2. Bosque tropical caducifolio en época de sequía de diciembre a mayo. Foto de Víctor J. Jaramillo, junio de 2013. animales que los conforman como a los seres humanos que se proveen de los bienes y servicios que se derivan de sus funciones, como son la obtención de alimento, agua, suelo fértil, plantas medicinales, madera y leña.

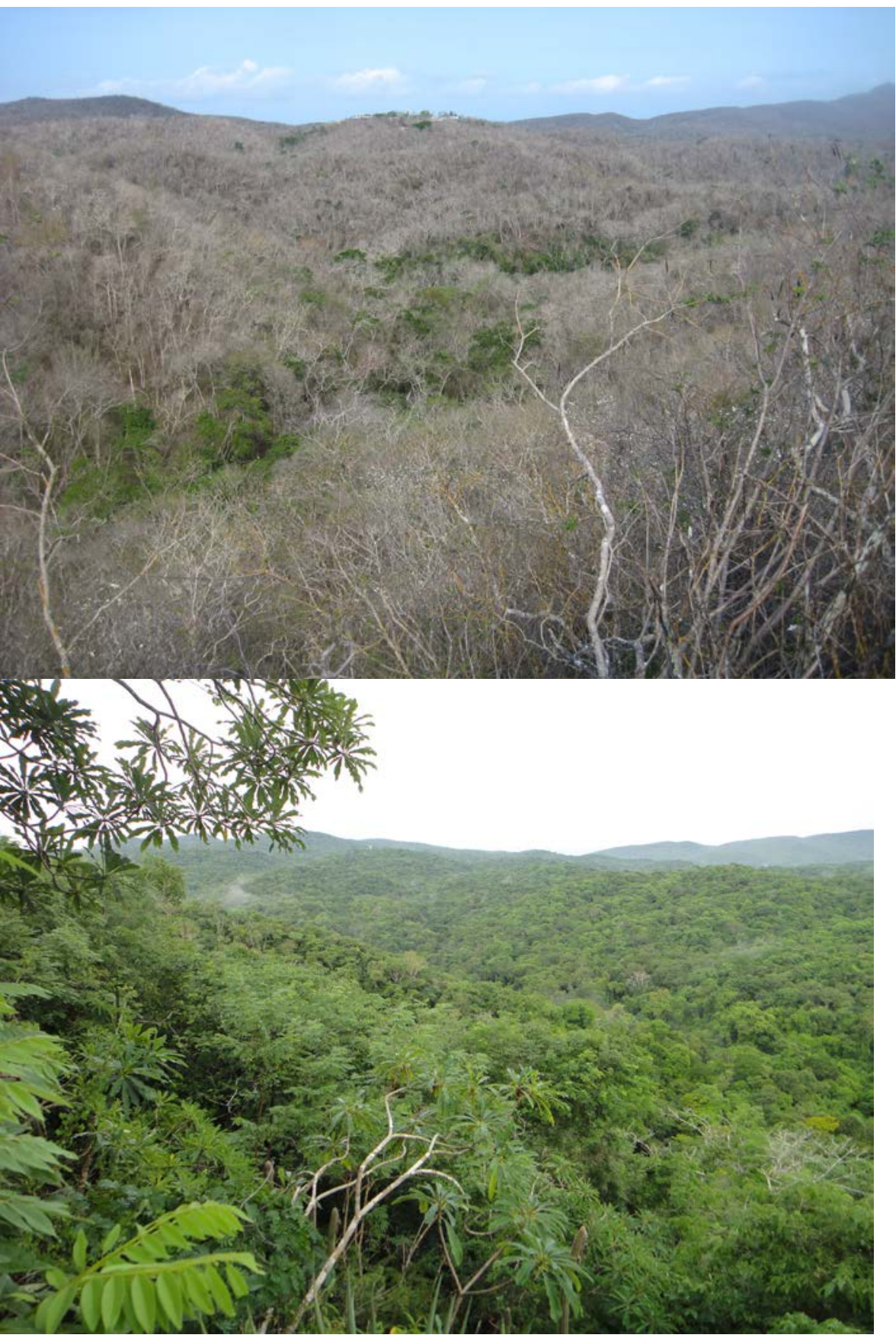

\section{La resiliencia del bosque tropical caducifolio}

La capacidad de los ecosistemas para recuperarse de perturbaciones naturales o causadas por los humanos, así como para permanecer en el tiempo se conoce como resiliencia. El estudio de esta característica ha recibido gran atención en la literatura científica, ya que es fundamental para entender cómo persisten los ecosistemas ante eventos extremos de perturbación.

Ubicado sobre la línea de costa del Pacífico mexicano y directamente expuesto al paso de huracanes se encuentra el bosque tropical caducifolio, así llamado porque la mayoría de los árboles tiran sus hojas en la época de sequía cada año (ver imágenes 2 y 3). En México, cubre grandes extensiones: desde el sur de Sonora y el suroeste de Chihuahua hasta Chiapas, e ingresa hacia el interior del país por las vertientes de los ríos Santiago y Balsas y hacia la Depresión Central de Chiapas, a través del Istmo de Tehuantepec. De acuerdo con el libro Vegetación de México de Rzedowski (1978), los árboles de estos bosques no son muy altos; generalmente alcanzan alturas de ocho a doce metros y coexisten con otras plantas de menor estatura como los arbustos o con plantas de troncos más delgados como las lianas o bejucos y con numerosas cactáceas y hierbas. El clima es cálido, con una temperatura que cambia muy poco a lo largo del año (de $22^{\circ} \mathrm{C}$ en promedio en enero a $26{ }^{\circ} \mathrm{C}$ en julio-agosto), pero con

Imagen 3. Bosque tropical caducifolio en época de lluvias de junio a noviembre. Foto de Manuel Maass, agosto de 2011.
Iluvias anuales que sí pueden variar mucho, desde escasas (400-600 mm) hasta abundantes (1,000 -1,300 mm). La lluvia cae normalmente entre junio y noviembre, pero en años excepcionales, como en los que se presenta el fenómeno climático conocido como El Niño, se presentan lluvias durante los 
meses invernales, que suelen ser secos. Entonces el bosque responde al producir nuevo follaje que pronto, ante la falta de agua, se vuelve a tirar.

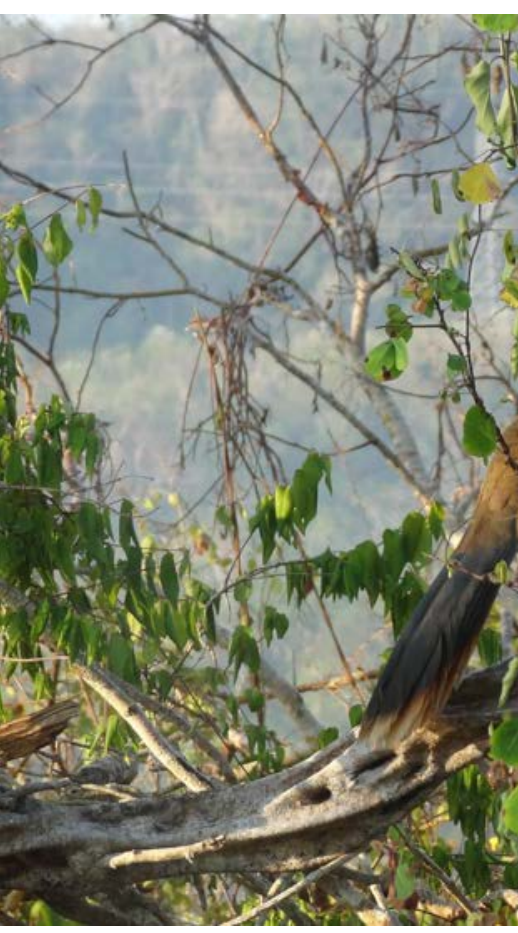

Imagen 4. Chachalaca, ave representativa del bosque tropical caducifolio. Foto de Manuel Maass, febrero, 2012.
¿Y cuál es la importancia biológica del bosque tropical caducifolio en nuestro país? La respuesta es que este bosque tiene un gran número de especies, tanto de plantas, con más de 4,500 especies (por ejemplo, barcino, copal chino, copal santo, tepeguaje, bonote, tetechos y candelabros), como de animales (armadillo, mapache, comadreja, tejón, puma, venado cola blanca, ocelote, jaguarundi y chachalaca, ver imagen 4). Además, muchas de ellas sólo se encuentran en este tipo de bosque tropical (casi la mitad de las especies de plantas y un tercio de las especies de vertebrados), lo que se conoce como especies endémicas. Tal es el caso de la codorniz cresta dorada, la rata arrocera y el murciélago platanero (Ceballos y García, 1995).

El ser humano ha mostrado su preferencia por ocupar estas áreas debido a la relativa facilidad para su aclareo, que es la remoción de los árboles con maquinaria o machete para darle un uso diferente a la tierra, por la fertilidad de los suelos y por su clima benigno. También, porque en estas zonas de clima seco la incidencia de enfermedades es menor cuando se le compara con las que se pueden contraer en las zonas de los bosques tropicales lluviosos. Todo esto ha provocado que exista una gran presión por parte de las comunidades que lo habitan para convertir el bosque a campos agrícolas y de pastoreo. Ello ha tenido como consecuencia que el bosque tropical caducifolio sea considerado un tipo de vegetación amenazado y altamente perturbado, tanto a nivel nacional como de manera global, donde se le conoce como bosque tropical seco.

\section{Los huracanes en la costa de Jalisco}

La transformación del ecosistema por acción humana, como la deforestación, no es la única amenaza a la integridad del bosque tropical caducifolio mexicano. La presencia de huracanes, como ya se mencionó, es un factor importante de perturbación porque llegan a causar una gran alteración no únicamente a los cultivos, a infraestructura de las comunidades y sus pobladores, sino a la vegetación nativa, dejando a su paso daños inmediatos visibles.

En octubre de 2011, la costa de Jalisco fue severamente afectada por el 
Cuadro 1. Fuente: CONAGUA, S.f.

Imagen 5. Imagen de satélite del Huracán Jova en 2011. Fuente: Comisión Nacional para el conocimiento y uso de la biodiversidad, National Aeronautics and Space Administration- Earth Observing System (2011) Imagen de satélite MODIS, 12 de octubre 2011. Resolución 1 km, bandas 4,3,1. México, Conabio. huracán Jova, de categoría 2, con vientos máximos sostenidos de 160 km por hora y rachas de hasta $195 \mathrm{~km}$ por hora (CONAGUA, 2011; ver cuadro 1, imagen 5), causando daños considerables al bosque en el área de Chamela-Cuixmala, ya que por ahí tocó tierra. Este huracán depositó en dos días una lluvia equivalente a 213 litros de agua en cada metro cuadrado de terreno $(213 \mathrm{~mm})$, cantidad casi 2.5 veces mayor a la que llueve normalmente durante todo el mes en años sin huracán.

\begin{tabular}{|c|c|c|c|c|c|c|}
\hline Nombre & $\begin{array}{c}\text { Año de } \\
\text { incidencia }\end{array}$ & $\begin{array}{l}\text { Categoría } \\
\text { máxima } \\
\text { alcanzada }\end{array}$ & $\begin{array}{c}\text { Vientos } \\
\text { máximos } \\
\text { sostenidos }\end{array}$ & $\begin{array}{l}\text { Costa } \\
\text { en la } \\
\text { que se } \\
\text { formó }\end{array}$ & $\begin{array}{l}\text { Período de } \\
\text { ocurrencia }\end{array}$ & $\begin{array}{c}\text { Estados } \\
\text { afectados } \\
\text { directamente }\end{array}$ \\
\hline Dean & 2007 & 5 & $\begin{array}{c}270 \mathrm{~km} / \mathrm{h} \\
\text { con rachas } \\
\text { de } 350 \\
\mathrm{~km} / \mathrm{h}\end{array}$ & Atlántico & $\begin{array}{c}13 \text { al } 23 \text { de } \\
\text { agosto }\end{array}$ & $\begin{array}{c}\text { Quintana } \\
\text { Roo, Yucatán, } \\
\text { Campeche, San } \\
\text { Luis Potosí, } \\
\text { Veracruz, Hidalgo, } \\
\text { Tamaulipas, } \\
\text { Tlaxcala, } \\
\text { Querétaro, } \\
\text { México, Nuevo } \\
\text { León, Distrito } \\
\text { Federal y alcanzó } \\
\text { la costa de Jalisco }\end{array}$ \\
\hline Jova & 2011 & 3 & $\begin{array}{c}205 \mathrm{~km} / \mathrm{h} \\
\text { con rachas } \\
\text { de } 250 \\
\mathrm{~km} / \mathrm{h}\end{array}$ & Pacífico & $\begin{array}{l}5 \text { al } 12 \text { de } \\
\text { octubre }\end{array}$ & $\begin{array}{c}\text { Michoacán, } \\
\text { Colima, Jalisco y } \\
\text { Nayarit }\end{array}$ \\
\hline Patricia & 2015 & 4 & $\begin{array}{l}325 \mathrm{~km} / \\
\text { hora, con } \\
\text { rachas de } \\
400 \mathrm{~km} / \mathrm{h}\end{array}$ & Pacífico & $\begin{array}{l}20 \text { al } 24 \text { de } \\
\text { octubre }\end{array}$ & $\begin{array}{l}\text { Nayarit, Jalisco, } \\
\text { Colima y } \\
\text { Michoacán }\end{array}$ \\
\hline
\end{tabular}

Tan solo cuatro años más tarde, en octubre de 2015, la zona fue nuevamente afectada por otro huracán, el huracán Patricia, considerado uno de los más intensos registrados en la historia moderna en el Pacífico (ver cuadro 1, imagen 6). Incluso, llegó a ser clasificado en la categoría 5, pero tocó tierra como categoría 4 en las costas de los municipios de la Huerta y Cihuatlán, en Jalisco. Los registros meteorológicos apuntaron a vientos máximos sostenidos, superiores a los del Jova, de 324 km por hora, con rachas de hasta 400 km por hora y lluvia muy copiosa, pero no tan abundante como la de Jova. Un fenómeno así no había ocurrido en la zona de ChamelaCuixmala en más de 60 años y mucho menos dos en un lapso tan corto. 
Imagen 6. Imagen de satélite del Huracán Patricia en el 2015. Fuente: Comisión Nacional para el conocimiento y uso de la biodiversidad, National Aeronautics and Space Administration- Earth Observing System (2015) Imagen de satélite MODIS, 23 de octubre 2015. Resolución 1 km, bandas 4,3,1. México, Conabio.

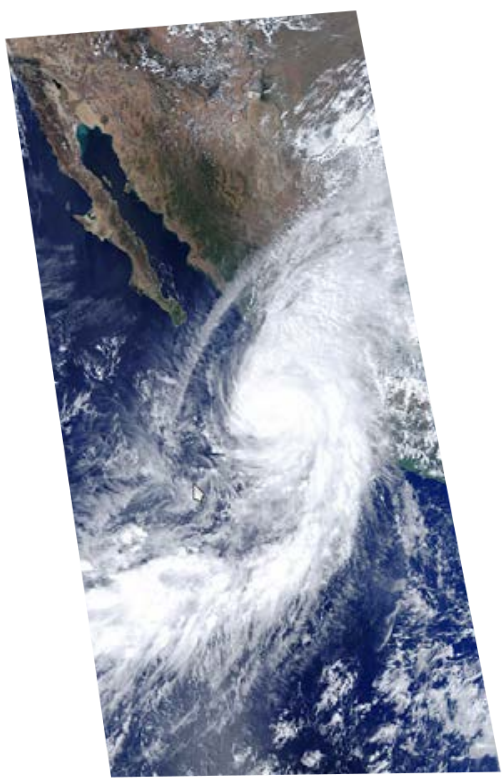

Las características de estos eventos hidrometeorológicos, como se les conoce a las tormentas tropicales y a los huracanes, en términos de la presencia de vientos de alta intensidad y de la cantidad de Iluvia depositada en unos cuantos días, rebasaron la variabilidad considerada como normal para la región. Su ocurrencia y la notable alteración que causan a la estructura y el funcionamiento del ecosistema plantean, entonces, una pregunta: ¿qué capacidad de recuperación, como expresión de su resiliencia, tiene el bosque de Chamela ante estos eventos? En virtud de la posibilidad de que aumente lafrecuencia de perturbaciones naturales asociadas al cambio climático, dicha capacidad adquirirá mayor relevancia, ya que de ella depende la provisión de un amplio conjunto de servicios para la sociedad entre los que se encuentran el suministro gratuito de madera, de leña y de productos no maderables, la conservación del suelo y de especies silvestres únicas de la región, así como la regulación del clima y de los ciclos de nutrientes.

\section{El funcionamiento del bosque y los estudios de largo plazo}

Las funciones que realiza el bosque están relacionadas con cuatro tipos de procesos ecológicos fundamentales: los del ciclo del agua, los ciclos de nutrientes, el flujo de energía y la dinámica de los grupos de organismos que viven juntos e interactúan entre sí en diferentes partes del bosque, conocidos como comunidades bióticas (conABIo, 2019). Dichos procesos, todos interconectados, pueden cambiar drásticamente en respuesta a las perturbaciones causadas por los huracanes, que, como ya vimos, modifican significativamente la cantidad, la calidad y la temporalidad de la lluvia. La alteración se manifiesta en importantes procesos como la cantidad de agua que se infiltra en el suelo y la que escurre hacia las partes bajas del terreno, en las cantidades de nutrientes disueltos en ella o adheridos al suelo que se erosiona, en la productividad del ecosistema y en las variaciones en la composición de especies y su distribución en el paisaje.

Los estudios de largo plazo que analizan estos procesos ecológicos por décadas proveen una herramienta científica fundamental para entender no sólo la variabilidad natural, sino también las alteraciones causadas en los ecosistemas por los huracanes, y para determinar su resiliencia, ya que proveen de información de las condiciones del bosque antes del disturbio y posterior a cada evento. En la Reserva de la Biósfera Chamela-Cuixmala, particularmente en la Estación de Biología Chamela, estamos realizando un estudio de largo 
plazo, con una base sólida de conocimientos generada a lo largo de más de 35 años, en cinco pequeñas cuencas hidrográficas (12-28 hectáreas) cubiertas por bosque tropical caducifolio sin perturbación de origen humano, en las que ha sido posible cuantificar los cuatro tipos de procesos ecológicos fundamentales. Hemos analizado la variación temporal de las entradas de agua (midiendo la precipitación o lluvia), así como las salidas (midiendo la cantidad que escurre) en la parte baja de cada una de las cuencas. Con el análisis químico del agua, la de la lluvia y la que escurre, hemos podido medir los nutrientes que circulan en el ecosistema, adheridos a partículas de suelo y materia orgánica o en forma disuelta en el agua. Tenemos también registros de la abundancia, crecimiento y mortalidad de los árboles, así como de la productividad del ecosistema y de su variación año con año.

Parte de la información generada por el proyecto sobre el impacto de los huracanes ya ha sido publicada en la literatura científica (ver por ejemplo, el número especial coordinado por Álvarez-Yépiz, Martínez-Yrízar y Fredericksen, 2018) que ha recibido una gran atención internacional, pues se han mostrado por primera vez, para el bosque tropical caducifolio en el Pacífico de México, los patrones de respuesta y de recuperación de diferentes procesos ecológicos ante esta singular combinación de eventos climáticos extremos. La información recabada hasta la fecha indica que de los últimos 33 años el más húmedo fue el 2015, cuando impactó el huracán Patricia con una precipitación de 1,329 mm y que fue cuatro veces más húmedo que el año más seco de toda la serie, el 2005, con solo 340 mm (Maass et al., 2018). Es decir, la variación en la cantidad de Iluvia que experimenta el bosque de Chamela puede ser muy grande. Esta alta variación también se debe a la mayor intensidad de la lluvia (la cantidad de agua por unidad de tiempo) y que curiosamente no la ha provocado, hasta ahora, un ciclón del Pacífico, sino uno originado en el Océano Atlántico: el huracán Dean. Este huracán depositó, en agosto de 2007, 236 mm de agua en menos de 24 horas, el equivalente a aproximadamente 12 garrafones de agua de 20 litros por cada metro cuadrado en un solo día. Se trata de una cantidad enorme de agua si consideramos que la lluvia producida por el huracán Patricia en un mismo período fue aproximadamente la mitad (115 mm). Que las lluvias generadas por Dean hayan alcanzado la costa del Pacífico habla de la vulnerabilidad y el riesgo al que está expuesto el país respecto a los huracanes, debido a la presencia de ambas costas y a la poca distancia que existe entre ellas en el centro y sur del territorio nacional. Este huracán ha sido considerado el más intenso registrado en la cuenca del Océano Atlántico, después del huracán Wilma, en el 2005.

Es importante señalar que, con las tormentas intensas, el agua que cae frecuentemente supera la capacidad del suelo para infiltrarla por lo que, al haber una pendiente, escurre sobre la superficie arrastrando material vegetal y sedimentos fuera del ecosistema. Estos procesos de escurrimiento se agravan durante los huracanes, que en el caso de Dean produjo en nuestras cuencas de estudio el mayor escurrimiento en el período de 33 años y también la mayor acumulación de sedimentos acarreados que fue casi seis veces superior a la 
producida por el huracán Patricia y nueve veces mayor a la de un año típico y sin la presencia de ciclones. Igualmente, la porción de nutrientes en solución que se movieron fuera de las cuencas, producto del escurrimiento asociado a los huracanes, fue también mayor, y en el caso del Jova, por ejemplo, duplicó las salidas de fósforo orgánico disuelto del ecosistema, en contraste con la cantidad que sale en años sin huracanes (Jaramillo et al., 2018). El fósforo orgánico representa uno de los constituyentes más importantes entre los nutrientes del suelo del bosque ya que es la forma en la que el fósforo puede ser aprovechado por los microorganismos del suelo y por las plantas. Entonces, su salida frecuente por eventos extremos como los ciclones puede representar una pérdida de fertilidad para el bosque a largo plazo. Esto habla del poder de los huracanes para alterar los procesos relacionados con los ciclos del agua y de los nutrientes en el bosque.

La actividad de los huracanes puede incorporar no sólo demasiada agua para el ecosistema, sino que se acompaña de fuertes vientos, los cuales causan la caída de material vegetal vivo al suelo y la muerte de árboles derribados por los vientos, como ocurrió con el huracán Patricia. El efecto instantáneo del viento de Jova y Patricia fue la caída masiva de hojas, ramitas, flores y frutos, conocidos en conjunto como hojarasca. La caída de hojarasca es un proceso clave del ecosistema, ya que representa una vía muy importante de transferencia de energía y nutrientes al suelo, al tiempo que es un indicador de la productividad primaria, la cual da soporte a las cadenas alimenticias del ecosistema. Nuestros estudios indican que este proceso fue fuertemente alterado por los dos huracanes. Las cantidades de hojarasca que cayeron durante los meses de octubre de 2011 y 2015, el mes del paso de los huracanes sobre la reserva de Chamela, superaron en casi cuatro y siete veces, respectivamente, el promedio de la hojarasca producida en el mismo mes en un período de 28 años previos a la entrada de estos dos huracanes (Martínez-Yrízar et al., 2018). Este pulso de caída de material vegetal tuvo un efecto en la cantidad de nutrientes que normalmente entran al suelo por esta vía. Por ejemplo, el nivel de nitrógeno y fósforo que regresó al suelo después del paso del huracán Patricia representó de dos a tres veces la cantidad total de dichos nutrientes que normalmente se reciben durante la temporada de lluvias completa (julio-octubre) en años sin huracán. De igual forma, las ramas y troncos derribados por los fuertes vientos de Patricia aumentaron la cantidad de material leñoso muerto, acumulado sobre la superficie del suelo del bosque en más de cincuenta toneladas por hectárea.

Lo anterior indica que los árboles del bosque de Chamela presentaron una baja resistencia a los fuertes vientos y a las grandes cantidades de lluvia provocadas por los huracanes, lo que cambió también de forma significativa la apariencia del bosque. Antes de Jova, el bosque medía $6.8 \mathrm{~m}$ de altura en promedio y era cerrado, con una muy baja fracción de claros (0.025 o 2.5\%) o aperturas en las copas de los árboles. Después del paso del huracán, el bosque perdió altura (5.9 m), se abrieron las copas de los árboles y el porcentaje de claros subió a 37.5\%. Cuatro años más tarde, después del huracán Patricia, el bosque disminuyó aún más en altura (3.5 
Con la información de largo plazo hemos podido mostrar que la incertidumbre en la cantidad y en la distribución estacional de la lluvia determinan lo que sucede y cómo sucede en este bosque. Reconocer esta incertidumbre e incorporarla en la toma de decisiones resulta esencial para establecer no sólo estrategias de conservación del bosque, sino también estrategias adaptativas y de manejo que disminuyan la vulnerabilidad de los habitantes de las regiones del trópico estacionalmente seco, en particular de los agricultores que dependen de los sistemas de producción agrícola de temporal. Es importante que gobernantes, líderes y la sociedad en general conozcan el valor de los bosques, estén conscientes de las amenazas asociadas a los huracanes y que estén preparados para responder ante tales contingencias.

\section{Referencias}

* Álvarez-Yépiz, Juan C., Martínez-Yrízar A. y Fredericksen, T. S. (2018, 15 de octubre). Special Issue: Resilience of Tropical Dry Forests to Extreme Disturbance Events. Forest Ecology and Management, 426:1-6. Dol: https://doi.org/10.1016/j. foreco.2018.05.067

* Ceballos, G. y A. García (1995). Conserving neotropical biodiversity: the role of dry forest in western Mexico. Conservation Biology, 9(6),1349-1356. https://www.jstor.org/stable/2387179

* Comisión Nacional para el Conocimiento y Uso de la Biodiverisdad (ConABIo). Procesos ecológicos. (2019, 13 de agosto). https://www.biodiversidad.gob.mx/ ecosistemas/procesose.html

* Comisión Nacional para el Conocimiento y uso de la Biodiversidad (ConABIo), National Aeronautics and Space Administration, Earth Observing System. (2011). Imagen de satélite MoDis, 12 de octubre 2011 [resolución 1 km, bandas 4,3,1].

* Comisión Nacional para el conocimiento y uso de la biodiversidad (CONABIO), National Aeronautics and Space Administration- Earth Observing System. (2015). Imagen de satélite modis, 23 de octubre 2015 [resolución 1 km, bandas 4,3,1].

- conagua. (s.f.). Consulta de historial y resumenes de ciclones tropicales. https://smn. conagua.gob.mx/es/ciclones-tropicales/informacion-historica

* encc. (2013). Estrategia Nacional de Cambio Climático. Visión 10-20-40. Gobierno de la República. https://www.gob.mx/cms/uploads/attachment/file/41978/ Estrategia-Nacional-Cambio-Climatico-2013.pdf

- Jaramillo, V., Martínez-Yrízar, A., Maass, M., Nava-Mendoza, M., Castañeda-Gómez, L., Ahedo-Hernández, R., Araiza, S., y Verduzco, A. (2018). Hurricane impact on biogeochemical processes in a tropical dry forest in western Mexico. Forest Ecology and Management 426:72-80. https://doi.org/10.1016/j.foreco.2017.12.031

- Maass, M., Ahedo-Hernández, R., Araiza, S., Verduzco, A., Martínez-Yrízar, A., Jaramillo, V., Parker, G., Pascual, F., García-Méndez, G., y Sarukhán, J. (2018). Long-term (33 years) rainfall and runoff dynamics in a tropical dry forest ecosystem in western Mexico: Management implications under extreme hydrometeorological. Events. Forest Ecology and Management 426:7-17. https://doi.org/10.1016/j.foreco.2017.09.040 
* Martínez-Yrízar, A., Jaramillo, V.J., Maass, M., Búrquez, A., Parker, G., Álvarez-Yépiz, J.C., Araiza, S., Verduzco, A., y Sarukhán, J. (2018). Resilience of tropical dry forest productivity to two hurricanes of different intensity in western Mexico. Forest Ecology and Management 426:53-60. DOI:10.1016/j.foreco.2018.02.024.

* Parker, G., Martínez-Yrízar, A., Álvarez-Yépiz, J.C., Maass, M., y Araiza, S. (2018). Effects of hurricane disturbance on a tropical dry forest canopy in western Mexico. Forest Ecology and Management 426:39-52. Dol: https://doi.org/10.1016/j. foreco.2017.11.037

* Programa Especial de Cambio Climático (PECC) 2014-2018. Logros 2016. (2016). Gobierno de la República. https://www.gob.mx/cms/uploads/attachment/ file/314952/Logros_PECC_2016.pdf

* Programa Especial de Cambio Climático (pecc) 2014-2018. (2014, 28 de abril). Diario Oficial de la Federación (DOF). http://www.sectur.gob.mx/wp-content/ uploads/2014/09/PECC-2014-2018.pdf

* Rzedowski, Jerzy. (1978). Vegetación de México. Editorial Limusa.

- Secretaría de Medio Ambiente y Recursos Naturales (semarnat) e Instituto Nacional de Ecología y Cambio Climático (INECC). (2012). Adaptación al cambio climático en México: visión, elementos y criterios para la toma de decisiones. Semarnat-InECC.

* Secretaría de Medio Ambiente y Recursos Naturales (semarnat). (2018). Informe de la Situación del Medio Ambiente en México. Compendio de Estadísticas Ambientales. Indicadores Clave, de Desempeño Ambiental y de Crecimiento Verde. Semarnat.

\section{Financiamiento}

El proyecto recibió financiamiento de PAPIIT-DGAPA-UNAM, PROYECTO No. IN209117, "Resiliencia del bosque tropical seco de Chamela ante el impacto de eventos climáticos extremos" y del conacrt, Proyecto 174094, "Respuesta del socioecosistema del bosque tropical seco de la región de Chamela al Huracán Jova: un evento catastrófico infrecuente".

\section{Cómo CITAR ESTE ARTículo}

* García-Méndez, Georgina, Jaramillo, V. J., Martínez-Yrízar, Angelina, Maass, Manuel, Sarukhán, José, Nava-Mendoza, Maribel, Ahedo, Raúl y Araiza, Salvador. (2020, septiembre-octubre). ¿Pueden los bosques tropicales resistir o recuperarse del impacto de los huracanes? Revista Digital Universitaria (RDU), 21(5). Dol: http://doi. org/10.22201/cuaieed.16076079e.2020.21.5.5 